\title{
openheart Prognostic impacts of changes in left ventricular ejection fraction in heart failure patients with preserved left ventricular ejection fraction
}

\author{
Akiomi Yoshihisa (10 , ${ }^{1,2}$ Yu Sato, ${ }^{1}$ Yuki Kanno, ${ }^{1}$ Mai Takiguchi, ${ }^{1}$ \\ Tetsuro Yokokawa, ${ }^{1,3}$ Satoshi Abe, ${ }^{1}$ Tomofumi Misaka, ${ }^{1,2}$ Takamasa Sato, ${ }^{1}$ \\ Masayoshi Oikawa, ${ }^{1}$ Atsushi Kobayashi, ${ }^{1}$ Takayoshi Yamaki, ${ }^{1}$ Hiroyuki Kunii, ${ }^{1}$ \\ Yasuchika Takeishi ${ }^{1}$
}

To cite: Yoshihisa A, Sato Y, Kanno Y, et al. Prognostic impacts of changes in left ventricular ejection fraction in heart failure patients with preserved left ventricular ejection fraction. Open Heart 2020;7:e001112. doi:10.1136/ openhrt-2019-001112

Received 12 June 2019 Revised 25 September 2019 Accepted 19 February 2020

Check for updates

(c) Author(s) (or their employer(s)) 2020. Re-use permitted under CC BY. Published by BMJ.

${ }^{1}$ Department of Cardiovascular Medicine, Fukushima Medical University, Fukushima, Japan ${ }^{2}$ Department of Advanced Cardiac Therapeutics, Fukushima Medical University, Fukushima, Japan ${ }^{3}$ Department of Pulmonary Hypertension, Fukushima Medical University, Fukushima, Japan

Correspondence to Dr Akiomi Yoshihisa; yoshihis@ fmu.ac.jp

\section{ABSTRACT}

Background It has been reported that recovery of left ventricular ejection fraction (LVEF) is associated with better prognosis in heart failure (HF) patients with reduced EF (rEF). However, change of LVEF has not yet been investigated in cases of HF with preserved EF (HFpEF). Methods and results Consecutive $1082 \mathrm{HFpEF}$ patients, who had been admitted to hospital due to decompensated $\mathrm{HF}$ (EF $>50 \%$ at the first LVEF assessment at discharge), were enrolled, and LVEF was reassessed within 6 months in the outpatient setting (second LVEF assessment). Among the HFpEF patients, LVEF of 758 patients remained above $50 \%$ (pEF group), 138 patients had LVEF of $40 \%-49 \%$ (midrange EF, mrEF group) and 186 patients had LVEF of less than $40 \%$ ( $\mathrm{rEF}$ group). In the multivariable logistic regression analysis, younger age and presence of higher levels of troponin I were predictors of rEF (worsened $\mathrm{HFpEF}$ ). In the Kaplan-Meier analysis, the cardiac event rate of the groups progressively increased from $\mathrm{DEF}, \mathrm{mrEF}$ to $\mathrm{rEF}$ (log-rank, $p<0.001$ ), whereas all-cause mortality did not significantly differ among the groups. In the multivariable Cox proportional hazard analysis, rEF (vs pEF) was not a predictor of all-cause mortality, but an independent predictor of increased cardiac event rates (HR $1.424,95 \% \mathrm{Cl} 1.020$ to $1.861, \mathrm{p}=0.039$ ).

Conclusion An initial assessment of LVEF and LVEF changes are important for deciding treatment and predicting prognosis in HFpEF patients. In addition, several confounding factors are associated with LVEF changes in worsened HFpEF patients.

\section{INTRODUCTION}

Left ventricular ejection fraction (LVEF) is among the most ingrained and commonly used quantities in clinical practice. LVEF is used in the diagnosis, characterisation, prognosis, patient triage and treatment selection of heart failure (HF) ${ }^{1-5}$ HF with reduced EF (HFrEF; LVEF $<40 \%$ ) is well characterised and established for evidencebased therapy, ${ }^{3-5}$ whereas HF with preserved

\section{Key questions}

What is already known about this subject?

- Changes in left ventricular ejection fraction (LVEF) and its prognostic impact on patients with heart failure with reduced $\mathrm{EF}$ (HFrEF) have recently been reported on; it has been suggested that the recovery of LVEF, known as 'recovered EF', occurs in a proportion of HFrEF patients, and is associated with better prognosis. However, LVEF changes, their clinical characteristics and prognostic impacts in $\mathrm{HF}$ patients with preserved EF (HFpEF) are unclear.

What does this study add?

- Of consecutive 1082 HFpEF patients, 186 (17.2\%) had LVEF of less than $40 \%$ at the second LVEF assessment (worsened HFpEF). Younger age, presence of coronary artery disease and sleep-disordered breathing, higher levels of troponin I and left ventricular end diastolic dimension were predictors of worsened HFpEF, which was associated with increased cardiac event rates.

How might this impact on clinical practice?

- An initial assessment of LVEF and LVEF changes are important for deciding treatment and predicting prognosis in HFpEF patients. In addition, several confounding factors are associated with LVEF changes in worsened HFpEF patients.

EF (HFpEF; LVEF $\geq 50 \%$ ) is a common and complex syndrome without evidence-based therapy. ${ }^{6}$ On the other hand, changes in LVEF and its prognostic impact on HFrEF patients have recently been reported on $^{8}$; it has been suggested that the recovery of EF, known as recovered EF, occurs in a proportion of HFrEF patients, and is associated with better prognosis. ${ }^{9-14}$ However, LVEF changes, their clinical characteristics and prognostic impacts in patients with HFpEF are unclear. 
Therefore, the aim of the current study was to clarify LVEF changes, their clinical characteristics and prognostic impacts in patients with HFpEF.

\section{METHODS}

This was a prospective observational study of 1161 decompensated HFpEF patients, who were discharged from Fukushima Medical University Hospital between 2010 and 2016, with LVEF $\geq 50 \%$ at discharge. The diagnosis of decompensated HF was made by several cardiologists based on the HF guidelines. ${ }^{3-5}$ Patients who had been admitted due to acute coronary syndrome and/or had previously undergone haemodialysis were excluded. All patients underwent echocardiography, and LVEF was assessed and HFpEF was determined at hospital discharge (first assessment), then premeditatedly reassessed in 1082 patients in the outpatient setting within 6 months (mean 3 months, range 2-6 months) postdischarge (second assessment). Of the 1161 patients, the second assessment was not performed in 75 , based on circumstances of the patients or the physicians, and four patients died or were hospitalised due to decompensated HF before the second assessment. We divided the remaining 1082 patients into three groups according to changes in LVEF observed at the second assessment: remained $\mathrm{pEF}$ (LVEF $\geq 50 \%, \mathrm{n}=758$ ); mid-range LVEF (mrEF) (LVEF 40\%-49\%, n=138) and reduced LVEF (rEF) (LVEF $<50 \%, \mathrm{n}=186)$.

We compared the patients' clinical features, laboratory data, echocardiography and ECG parameters, and postdischarge prognosis. The patients were followed up until 2018 for cardiac events and all-cause death. Cardiac events were defined as worsened HF and cardiac death. Cardiac death was classified by independent experienced cardiologists as death from worsened HF, ventricular fibrillation documented by ECG or implantable devices, or acute coronary syndrome. Worsened HF was defined as hospitalisation due to decompensated HF. Postdischarge, the patients visited our hospital or their referring hospital once every 1-2 months. Status and dates of death were obtained from the patients' medical records. If these data were unavailable, status was ascertained by a telephone call to the patient's referring hospital physician. We were able to follow up on all patients who had undergone the second assessment. Those administering the survey were blind to the analyses, and written informed consent was obtained from all study subjects. $^{15}$

We evaluated several comorbidities that often coexist and are associated with adverse prognosis in HF patients. ${ }^{16}$ Coronary artery disease $(\mathrm{CAD})$ was confirmed by the following: myocardial scintigraphy, coronary CT angiography and/ or coronary angiography. Atrial fibrillation (AF) was identified by ECG performed during hospitalisation and/or from medical records. Hypertension was defined as the recent use of antihypertensive drugs, systolic blood pressure $\geq 140 \mathrm{~mm} \mathrm{Hg}$ and/or diastolic blood pressure $\geq 90 \mathrm{~mm}$ $\mathrm{Hg}$. Diabetes mellitus was defined as the recent use of antidiabetic drugs, a fasting glucose value of $\geq 126 \mathrm{mg} / \mathrm{dL}$, a casual glucose value of $\geq 200 \mathrm{mg} / \mathrm{dL}$ and/or $\mathrm{HbA1c} \geq 6.5 \%$ (National Glycohemoglobin Standardization Program). Dyslipidaemia was defined as the recent use of cholesterollowering drugs, a triglyceride value of $\geq 150 \mathrm{mg} / \mathrm{dL}$, a lowdensity lipoprotein cholesterol value of $\geq 140 \mathrm{mg} / \mathrm{dL}$ and/ or a high-density lipoprotein cholesterol value of $<40 \mathrm{mg} /$ dL. Chronic kidney disease (CKD) was defined as an estimated glomerular filtration rate of $<60 \mathrm{~mL} / \mathrm{min} / 1.73 \mathrm{~m}^{2}$ according to the Modification of Diet in Renal Disease formula. ${ }^{17} 18$ Anaemia was defined as haemoglobin levels of $<12.0 \mathrm{~g} / \mathrm{dL}$ in females and $<13.0 \mathrm{~g} / \mathrm{dL}$ in males. ${ }^{5}$ Hyperuricaemia was defined as regular usage of antihyperuricemic agents or serum uric acid levels of over $7 \mathrm{mg} / \mathrm{dL}^{19}$ Sleep-disordered breathing (SDB) was defined as apnoeahypopnoea index of $>5$ times/hour, and included both central and obstructive SDB, determined by a portable sleep monitor, polysomnography and/or from medical records. ${ }^{20-22}$ Chronic obstructive pulmonary disease was defined as forced expiratory volume in one second/forced vital capacity of $<70 \%$ by spirometry according to the Global Initiative for Chronic Obstructive Lung Disease, the American Thoracic Society/European Respiratory Society guidelines, and/or from medical records. ${ }^{23}$ Peripheral artery disease was diagnosed according to the recent guidelines using CT, angiography and/or ankle-brachial index. ${ }^{24}{ }^{25}$

\section{Measurement of parameters of laboratory data, ECG and echocardiography}

Blood samples were obtained from all patients at Fukushima Medical University Hospital at hospital discharge. B-type natriuretic peptide (BNP) levels were measured using a specific immunoradiometric assay (Shionoria BNP kit, Shionogi, Osaka, Japan). High-sensitivity troponin I levels were measured using EDTA anticoagulated plasma with a refined assay (Abbott-Architect, Abbott Laboratories, Abbott Park, Illinois, USA).

The standard resting ECG was recorded in the supine position with CardioStar FCP-7541 (Fukuda Denshi, Tokyo, Japan) and stored digitally. This system allows automatic measuring of QT and QTc interval. The QT interval was measured from the beginning of the QRS complex until the $\mathrm{T}$ wave returned to the isoelectric line. The median QT interval was then calculated and corrected for the heart rate. $^{18}$

Echocardiography was performed blindly by experienced echocardiographers using standard techniques. ${ }^{16} 23$ The echocardiographic parameters investigated included left ventricular diastolic dimension (LVDd), left ventricular systolic dimension (LVDs), LVEF, left atrium volume, ratio of early transmitral flow velocity to mitral annular velocity (mitral valve $\mathrm{E} / \mathrm{e}$ '), inferior vena cava diameter (IVC), tricuspid regurgitation pressure gradient (TR-PG) and right ventricular fractional area change (RV-FAC). ${ }^{26}$ The LVEF was calculated using Simpson's method in a four-chamber view. ${ }^{16}{ }^{23}$ The intraobserver variability (the SD of the differences/average value) of LVEF was $6 \% \pm 2 \%$. The RV-FAC, defined as (end-diastolic area and end-systolic area)/end diastolic area $\times 100$, was used as a measure of right ventricular 
systolic function. All measurements were performed using ultrasound systems (ACUSON Sequoia, Siemens Medical Solutions USA, Mountain View, California, USA).

\section{Statistical analysis}

Categorical variables are expressed as numbers and percentages. $\mathrm{A} \mathrm{X}^{2}$ test was used for comparisons of categorical variables, followed by Fisher's exact test when appropriate. Normality was confirmed using the Shapiro-Wilk test in each group. Parametric variables are presented as

\begin{tabular}{|c|c|c|c|c|}
\hline & pEF (=758) & mrEF (=138) & rEF (=186) & $P$ value \\
\hline Age (years) & $67.8 \pm 14.5$ & $67.5 \pm 13.2$ & $65.2 \pm 15.8$ & 0.086 \\
\hline Male gender (n, \%) & $404(53.3)$ & $87(63.0)$ & $123(66.1)$ & 0.002 \\
\hline Body mass index $\left(\mathrm{kg} / \mathrm{m}^{2}\right)$ & $23.5 \pm 4.3$ & $23.1 \pm 3.8$ & $22.6 \pm 3.6^{*}$ & 0.025 \\
\hline Systolic blood pressure (mm Hg) & $130.4 \pm 30.2$ & $135.2 \pm 30.8$ & $130.1 \pm 33.3$ & 0.223 \\
\hline Diastolic blood pressure (mm Hg) & $72.8 \pm 31.2$ & $74.7 \pm 24.5$ & $74.8 \pm 22.9$ & 0.610 \\
\hline Heart rate (bpm) & $75.5 \pm 23.8$ & $77.5 \pm 24.6^{\star \star}$ & $86.6 \pm 25.3^{\star \star}$ & $<0.001$ \\
\hline NYHA functional class III/IV (n, \%) & $26(3.4)$ & $2(1.4)$ & $7(3.8)$ & 0.435 \\
\hline \multicolumn{5}{|l|}{ Comorbidity } \\
\hline Coronary artery disease $(n, \%)$ & $187(24.7)$ & $44(31.9)$ & $78(41.9)$ & $<0.001$ \\
\hline Atrial fibrillation (n, \%) & $290(38.3)$ & $64(46.4)$ & $86(46.2)$ & 0.048 \\
\hline Hypertension (n, \%) & $521(68.7)$ & $94(68.1)$ & $140(75.3)$ & 0.199 \\
\hline Diabetes $(\mathrm{n}, \%)$ & $248(32.7)$ & $51(37.0)$ & $83(44.8)$ & 0.009 \\
\hline Dyslipidaemia $(n, \%)$ & $524(69.1)$ & $113(81.9)$ & $134(72.0)$ & 0.009 \\
\hline Chronic kidney disease $(\mathrm{n}, \%)$ & $353(46.6)$ & $80(58.0)$ & $109(58.6)$ & 0.002 \\
\hline Anaemia $(n, \%)$ & $381(50.3)$ & $82(59.4)$ & $120(64.5)$ & 0.001 \\
\hline Hyperurecaemia (n, \%) & $375(49.5)$ & $92(66.7)$ & $135(72.6)$ & $<0.001$ \\
\hline Sleep-disordered breathing $(n, \%)$ & $253(33.4)$ & $62(44.9)$ & $99(53.2)$ & $<0.001$ \\
\hline COPD (n, \%) & $171(22.6)$ & $35(25.4)$ & $51(27.4)$ & 0.337 \\
\hline Peripheral artery disease (n, \%) & $67(8.8)$ & $12(8.7)$ & $25(13.4)$ & 0.150 \\
\hline Smoking (n, \%) & $385(51.7)$ & $78(58.2)$ & $95(51.4)$ & 0.359 \\
\hline Alcohol (n, \%) & $69(9.3)$ & $16(11.9)$ & $12(6.5)$ & 0.240 \\
\hline \multicolumn{5}{|l|}{ Treatment } \\
\hline RAS inhibitor (n, \%) & $484(63.9)$ & $97(70.3)$ & $146(78.5)$ & $<0.001$ \\
\hline Mineral receptor antagonist $(\mathrm{n}, \%)$ & $215(28.4)$ & $54(39.1)$ & $101(54.3)$ & $<0.001$ \\
\hline Calcium channel blocker (n, \%) & $322(42.5)$ & $54(39.1)$ & $73(39.2)$ & 0.604 \\
\hline Beta blocker (n, \%) & $440(58.0)$ & $111(80.4)$ & $155(83.3)$ & $<0.001$ \\
\hline Diuretic (n, \%) & $415(54.7)$ & $93(67.4)$ & $147(79.0)$ & $<0.001$ \\
\hline Statin (n, \%) & $295(39.6)$ & $56(41.8)$ & $75(40.5)$ & 0.882 \\
\hline Digitalis (n, \%) & $86(11.5)$ & $11(8.2)$ & $21(11.4)$ & 0.523 \\
\hline Amiodarone (n, \%) & $55(7.3)$ & $14(10.1)$ & $36(19.4)$ & $<0.001$ \\
\hline Antiplatelet agent (n, \%) & $353(46.6)$ & $83(60.1)$ & $114(61.3)$ & $<0.001$ \\
\hline Anticoagulant (n, \%) & $396(52.2)$ & $95(68.8)$ & $126(67.7)$ & $<0.001$ \\
\hline $\mathrm{PCl}(\mathrm{n}, \%)$ & $123(16.2)$ & $40(29.0)$ & $59(31.7)$ & $<0.001$ \\
\hline Catheter ablation (n, \%) & $82(10.8)$ & $10(7.2)$ & $19(10.2)$ & 0.445 \\
\hline $\operatorname{ICD}(\mathrm{n}, \%)$ & $91(12.2)$ & $16(11.9)$ & $26(14.1)$ & 0.778 \\
\hline
\end{tabular}


mean $\pm \mathrm{SD}$ and non-parametric variables $(\mathrm{eg}, \mathrm{BNP}$, troponin $\mathrm{I}$ and $\mathrm{C}$ reactive protein) are presented as a median and IQR. Parametric variables were compared using analysis of variance (ANOVA), and equality was tested using the Levene test. If the data were equal, ANOVA was followed by Tukey's honest significant difference. If the data were not equal, the Games-Howell post hoc test was used. Non-parametric variables were compared using the Kruskal-Wallis test. We performed logistic regression analysis allowing for interaction between the onset of rEF; worsened HFpEF and each possible confounding factor. Kaplan-Meier analysis was used for presenting the cardiac event rate and allcause mortality, and the log-rank test was used for initial comparisons. The Kaplan-Meier estimates of the survival curves were plotted against time to follow-up period. These curves helped in identifying non-proportionality patterns in hazard function such as convergence (difference in risk between the groups decreases with time), divergence or crossing of the curves. In addition, a Schoenfeld test for the violation of proportional hazards, which can be used to assess the correlation between scaled residuals and time, was also conducted. Univariable and multivariable

Table 2 Laboratory and echocardiographic data of patients with HFpEF at first LVEF assessment, and whose changes in LVEF at second assessment $(n=1082)$

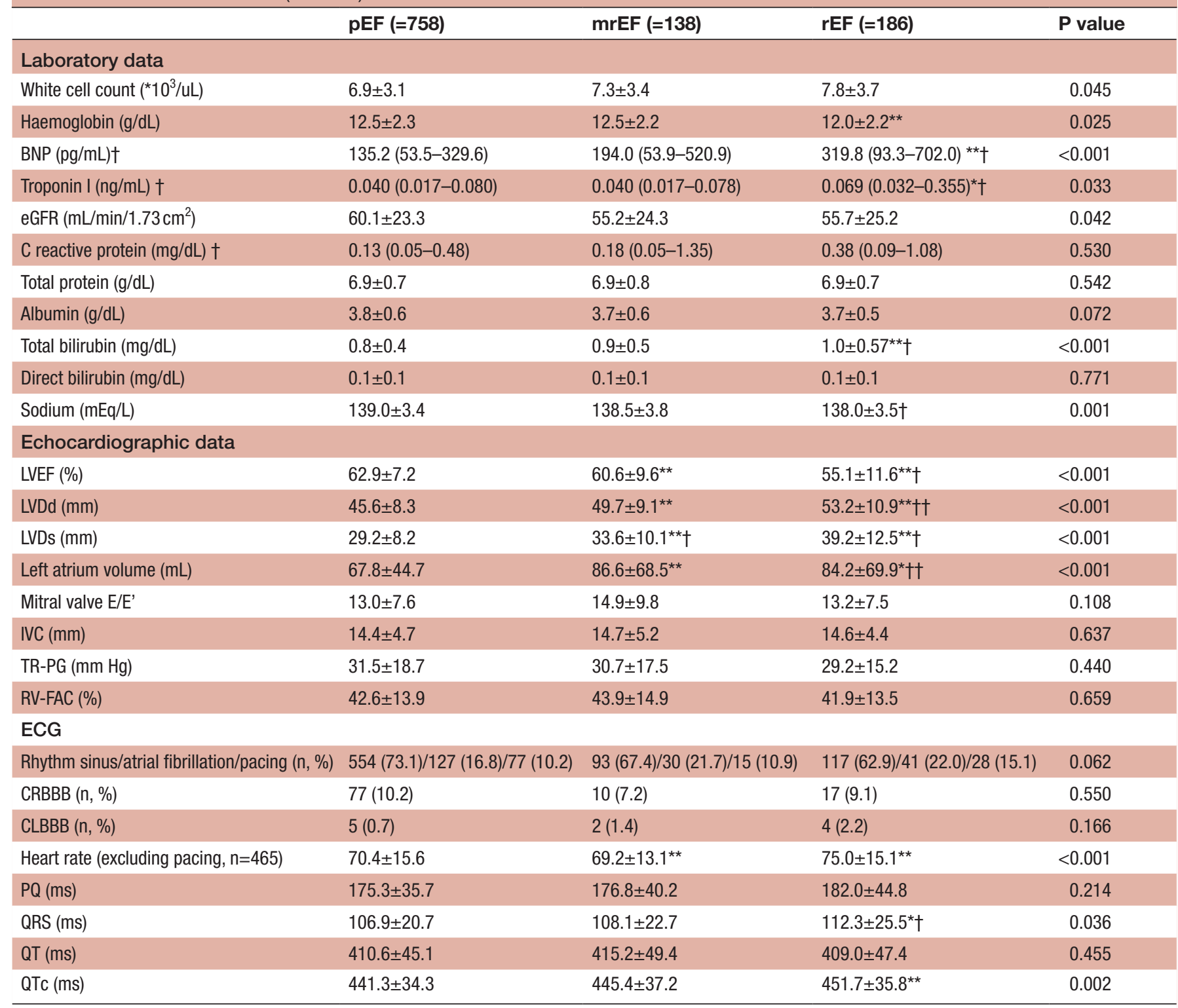

${ }^{*} \mathrm{P}<0.05,{ }^{*} \mathrm{P}<0.01$ vs $\mathrm{pEF}, \mathrm{\dagger p}<0.05$ and $\dagger \dagger \mathrm{p}<0.01$ vs $\mathrm{mrEF}$.

†Data are presented as median (IQR).

BNP, B-type natriuretic peptide; CLBBB, complete left bundle branch block; CRBBB, complete right bundle branch block; GFR, glomerular filtration rate; HFpEF, heart failure with preserved LVEF; IVC, inferior vena cava diameter; LVDd, left ventricular end diastolic dimension; LVDs, left ventricular end systolic dimension; LVEF, left ventricular ejection fraction; mrEF, mid-range LVEF at second assessment; $p E F$, remained preserved LVEF at second assessment; rEF, reduced LVEF at second assessment; RV-FAC, right ventricular fractional area change; TR-PG, tricuspid regurgitation pressure gradient. 
Heart failure and cardiomyopathies

Table 3 Logistic regression analysis: associations between the clinical profiles and 'rEF at second LVEF assessment'

\begin{tabular}{|c|c|c|c|c|c|c|}
\hline & Univa & & & Multi & & \\
\hline & OR & $95 \% \mathrm{Cl}$ & $P$ value & OR & $95 \% \mathrm{Cl}$ & $P$ value \\
\hline Age & 0.988 & 0.978 to 0.999 & 0.028 & 0.974 & 0.953 to 0.995 & 0.016 \\
\hline Male gender & 1.610 & 1.157 to 2.242 & 0.005 & 0.998 & 0.505 to 1.971 & 0.995 \\
\hline Body mass index & 0.948 & 0.909 to 0.988 & 0.012 & 0.933 & 0.865 to 1.007 & 0.073 \\
\hline Systolic blood pressure & 0.999 & 0.994 to 1.004 & 0.666 & & & \\
\hline Heart rate & 1.016 & 1.010 to 1.022 & $<0.001$ & 1.012 & 0.996 to 1.029 & 0.138 \\
\hline NYHA class III or IV & 1.212 & 0.521 to 2.819 & 0.655 & & & \\
\hline Coronary artery disease & 2.079 & 1.499 to 2.885 & $<0.001$ & 2.112 & 1.047 to 4.259 & 0.037 \\
\hline Atrial fibrillation & 1.317 & 0.958 to 1.809 & 0.090 & & & \\
\hline Hypertension & 1.391 & 0.968 to 1.997 & 0.074 & & & \\
\hline Diabetes & 1.609 & 1.168 to 2.217 & 0.004 & 1.517 & 0.789 to 2.915 & 0.211 \\
\hline Dyslipidaemia & 1.048 & 0.739 to 1.489 & 0.795 & & & \\
\hline Chronic kidney disease & 1.514 & 1.099 to 2.084 & 0.011 & 0.694 & 0.342 to 1.409 & 0.312 \\
\hline Anaemia & 1.700 & 1.225 to 2.360 & 0.001 & 1.378 & 0.699 to 2.719 & 0.355 \\
\hline Hyperuricaemia & 2.432 & 1.717 to 3.443 & $<0.001$ & 1.372 & 0.681 to 2.762 & 0.376 \\
\hline Sleep-disordered breathing & 2.099 & 1.526 to 2.888 & $<0.001$ & 1.212 & 1.007 to 1.472 & 0.042 \\
\hline COPD & 1.265 & 0.885 to 1.810 & 0.197 & & & \\
\hline Peripheral artery disease & 1.606 & 0.993 to 2.596 & 0.093 & & & \\
\hline Smoking & 0.948 & 0.691 to 1.302 & 0.743 & & & \\
\hline Alcohol & 0.648 & 0.346 to 1.212 & 0.175 & & & \\
\hline Log BNP & 2.600 & 1.864 to 3.628 & $<0.001$ & 1.543 & 0.845 to 2.817 & 0.158 \\
\hline Log troponin I & 1.465 & 1.222 to 1.756 & $<0.001$ & 1.574 & 1.055 to 2.349 & 0.026 \\
\hline LVDd & 1.080 & 1.059 to 1.101 & $<0.001$ & 1.069 & 1.030 to 1.109 & $<0.001$ \\
\hline LVEF & 0.908 & 0.888 to 0.927 & $<0.001$ & 0.933 & 0.902 to 0.966 & $<0.001$ \\
\hline CLBBB & 2.791 & 0.809 to 9.634 & 0.104 & & & \\
\hline QRS & 1.008 & 1.001 to 1.015 & 0.026 & 0.997 & 0.983 to 1.011 & 0.662 \\
\hline QT & 0.999 & 0.995 to 1.003 & 0.559 & & & \\
\hline QTC & 1.008 & 1.003 to 1.012 & 0.002 & 1.004 & 0.995 to 1.013 & 0.409 \\
\hline RAS inhibitors & 0.725 & 0.547 to 0.936 & 0.037 & 0.931 & 0.472 to 1.388 & 0.281 \\
\hline Mineral receptor antagonist & 0.926 & 0.562 to 1.324 & 0.463 & & & \\
\hline Calcium channel blocker & 0.893 & 0.647 to 1.234 & 0.494 & & & \\
\hline Beta blocker & 0.892 & 0.413 to 1.326 & 0.782 & & & \\
\hline Diuretic & 2.879 & 1.975 to 4.197 & $<0.001$ & 2.272 & 0.970 to 5.320 & 0.414 \\
\hline Statin & 1.297 & 0.943 to 1.783 & 0.110 & & & \\
\hline Digitalis & 1.501 & 0.915 to 2.462 & 0.108 & & & \\
\hline ICD & 1.180 & 0.744 to 1.871 & 0.482 & & & \\
\hline
\end{tabular}

BNP, B-type natriuretic peptide; CLBBB, complete left bundle branch block; COPD, chronic obstructive pulmonary disease; HFpEF, heart failure with preserved LVEF; ICD, implantable cardiac defibrillator; LVDd, left ventricular end diastolic dimension; LVEF, left ventricular ejection fraction; LVEF, left ventricular ejection fraction; NYHA, New York Heart Association; RAS, renin-angiotensin-aldosterone system; rEF, reduced LVEF at second assessment.

Cox proportional hazard analyses were used to evaluate changes of LVEF as a predictor of cardiac event rates and all-cause mortality. Univariable parameters with $\mathrm{p}<0.05$ were included in the multivariable analysis. The proportional hazards assumption for the model was checked by examining log minus-log transformed data. A $\mathrm{p}<0.05$ was considered statistically significant for all comparisons, and all analyses were performed using a statistical software package (SPSS V.24.0).

\section{RESULTS}

The clinical characteristics of patients who underwent the second LVEF assessment are presented in table 1 . The rEF group had a lower body mass index and higher 

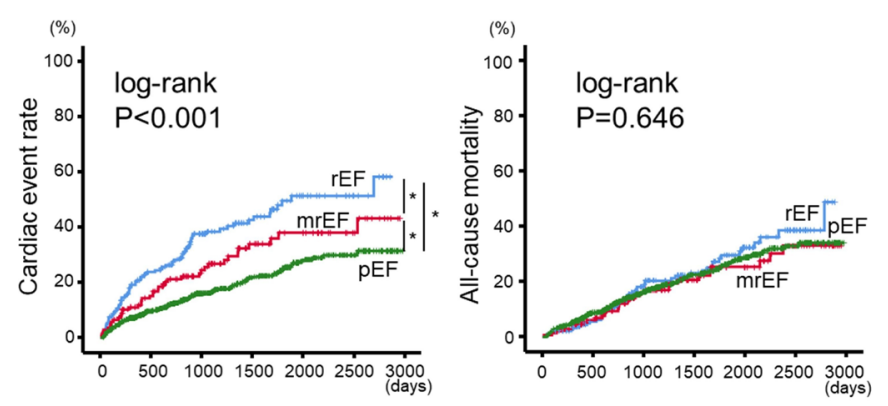

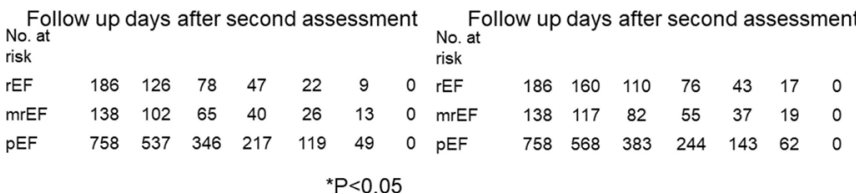

Figure 1 Rates of cardiac events and all-cause mortality with changes in left ventricular ejection fraction (LVEF) in heart failure patients with preserved LVEF (HFpEF). KaplanMeier analysis, during the follow-up period after the second assessment of LVEF, for cardiac event rate and all-cause mortality based on changes in LVEF between the first and second assessments. remained preserved LVEF (pEF) (LVEF $\geq 50 \%, n=758$ ); mid-range LVEF (mrEF) (LVEF 40\%-49\%, $\mathrm{n}=138)$; and reduced LVEF (rEF) (LVEF $<50 \%, \mathrm{n}=186)$ at the second assessment.

Table 4 Cox proportional hazard model of cardiac events and all-cause mortality in HFpEF

\begin{tabular}{lllr}
\hline & HR & 95\% Cl & P value \\
\hline $\begin{array}{l}\text { Cardiac event } \\
\text { (252 events/1082 } \\
\text { patients) }\end{array}$ & & & \\
pEF & Ref & & \\
mrEF & 1.593 & 1.119 to 2.266 & 0.010 \\
\hline mrEF adjusted* & 1.185 & 0.728 to 1.732 & 0.543 \\
\hline rEF 'worsened HFpEF' & 2.439 & 1.842 to 3.230 & $<0.001$ \\
\hline rEF 'worsened HFpEF' & 1.424 & 1.020 to 1.861 & 0.039 \\
adjusted* & & & \\
\hline
\end{tabular}

\begin{tabular}{llll}
$\begin{array}{l}\text { All-cause mortality } \\
\text { (226 events/1082 } \\
\text { patients) }\end{array}$ & & & \\
pEF & Ref & & \\
mrEF & 0.925 & 0.621 to 1.377 & 0.699 \\
\hline rEF 'worsened HFpEF' & 1.134 & 0.819 to 1.570 & 0.450 \\
\hline
\end{tabular}

${ }^{*}$ Adjusted: adjusted for age, gender, body mass index, systolic blood pressure, heart rate, New York Heart Association class III or IV, presence of coronary artery disease, atrial fibrillation, hypertension, diabetes, dyslipidaemia, chronic kidney disease, anaemia, hyperuricaemia, sleep-disordered breathing, chronic obstructive pulmonary disease, smoking, alcohol, usage of renin-angiotensin-aldosterone system inhibitors, mineral receptor antagonist, calcium channel blocker, beta blockers, diuretics, statin, digitalis, implantable cardiac defibrillator, B-type natriuretic peptide, tricuspid regurgitation pressure gradient, right ventricular fractional area change, mitral regurgitation, left atrium volume and left ventricular ejection fraction at first assessment.

$\mathrm{HFpEF}$, heart failure with preserved LVEF; mrEF, mid-range LVEF at second assessment; $p E F$, remained preserved LVEF at second assessment; rEF, reduced LVEF at second assessment. heart rate when compared with the $\mathrm{pEF}$ group. In addition, the prevalences of male gender, $\mathrm{CAD}$, diabetes, $\mathrm{CKD}$, anaemia, hyperuricaemia and SDB were highest in the rEF group among the groups. The findings of laboratory data, ECG and echocardiography are presented in table 2. BNP, troponin I, total bilirubin, LVDd, LVDs and QRS were highest, and LVEF was lowest in the rEF group. In contrast, other parameters, including $\mathrm{C}$ reactive protein, total protein, mitral valve E/E', IVC, TR-PG, RV-FAC, PQ and QT, did not significantly differ among the groups. In the multivariable logistic regression analysis (table 3), younger age, presence of CAD and SDB, higher levels of troponin I, LVDd, and lower levels of $\mathrm{LVEF}$ at the first assessment were predictors of rEF (worsened HFpEF).

During the follow-up period (mean $1228 \pm 790$ days, range 15-2975 days), 252 cardiac events, including 218 hospitalisations due to $\mathrm{HF}$ and 34 cardiac deaths, occurred, as well as 226 all-cause mortalities (91 cardiac deaths and 135 non-cardiac deaths). In the Kaplan-Meier analysis (figure 1), the cardiac event rate of the groups progressively increased from $\mathrm{pEF}$, mrEF to rEF (log-rank, $\mathrm{p}<0.001)$, whereas all-cause mortality did not significantly differ among the groups. In the univariable and multivariable Cox proportional hazard analyses (table 4), rEF (vs $\mathrm{pEF}$ ) was not a predictor of all-cause mortality, but an independent predictor of increased cardiac event rates after adjustment of LVEF at the first assessment (HR $1.424,95 \%$ CI 1.020 to $1.861, \mathrm{p}=0.039$ ).

\section{DISCUSSION}

In the present study, we demonstrated that patients with rEF; worsened HFpEF, which was $17.2 \%$ in the present study, were associated with younger age, higher presence of several comorbidities, including CAD and SDB, and higher levels of troponin I and LVDd, indicating the presence of myocardial damage and structural remodelling, and worse cardiac event rate. However, all-cause mortality did not necessarily differ with LVEF changes in the HFpEF patients.

It has been reported that there were differences in prognostic factors between HFrEF and HFpEF. ${ }^{27}{ }^{28}$ Older age and diabetes mellitus were predictors of HFrEF and $\mathrm{HFpEF}$, and higher body mass index and $\mathrm{AF}$ were predictors of HFpEF, whereas male gender, higher heart rate, hypertension, cardiovascular disease, left ventricular hypertrophy and left bundle-branch block were predictors of HFrEF risk. ${ }^{29} 30$

Although LVEF assessment is generally used to predict the prognosis and select the treatment for $\mathrm{HF}^{1-5} \mathrm{LVEF}$ changes and their prognostic impacts on HFrEF, have recently been reported as 'recovered EF' ${ }^{8-14}$ However, there are few reports on the prognostic impact of LVEF changes in HFpEF patients. In the present study, worsened HFpEF was not a predictor of all-cause mortality, but an independent predictor of increased cardiac event rates after adjustment for baseline LVEF. Thus, not only 
baseline LVEF, but also its changes, seem to be associated with cardiac event rates in HFpEF patients. Tsuji $e t$ al reported that worsened HFpEF was observed in only $1.9 \%$ of stable HFpEF patients over a 1-year period, and was associated with higher all-cause mortality compared with patients with persistent HFpEF. ${ }^{31}$ Dunlay et al reported that EF progressively decreases with ageing in HF patients, and that a decrease in LVEF was associated with prevalence of CAD, as well as reduced survival. ${ }^{32}$

Male gender, ${ }^{8} \mathrm{CAD},{ }^{63} \mathrm{AF}^{34}$ diabetes, ${ }^{633} 35 \mathrm{CKD},{ }^{633} 35$ anaemia, ${ }^{33}$ hyperuricaemia ${ }^{19}$ and SDB $^{35-37}$ have been reported to be associated with left ventricular remodelling and adverse prognosis in HF patients. However, younger age, non-ischaemic aetiology and fewer comorbidities are associated with left ventricular reverse remodelling in HF patients. ${ }^{2}$ In particular, compared with HFrEF, HFpEF has many comorbidities, which contribute to HF progression. ${ }^{16}$ LVEF itself is not necessarily associated with mortality, and non-cardiac comorbidity has a greater prognostic impact on HFpEF than HFrEF. ${ }^{28} 38$ Concordant with these findings, ${ }^{28} 38$ in the present study, non-cardiac mortality was higher than cardiac mortality in HFpEF patients.

\section{Study strengths and limitations}

There are several strengths to our study. This is the first study to show changes in LVEF, comprehensive confounding factors for changes in LVEF and their prognostic impacts in HFpEF patients.

The present study also has several limitations. First, as a prospective cohort study of a single centre with a relatively small number of patients, the present results may not be representative of the general population. Second, we could not examine all patients, who had undergone the first assessment LVEF, at the second assessment (93.2\%) because of losing follow-up and/or occurrence of event before the second assessment, and selection bias could not be fully denied. Although LVEF was reassessed in the outpatient setting within half a year, the time periods between the first and second assessments differ from patient to patient. Third, the present study included only variables relating to hospitalisation for decompensated HF, and we did not take into consideration changes in medical parameters or treatments, other than LVEF. Therefore, the present results should be viewed as preliminary, and further studies with larger populations are needed.

\section{CONCLUSIONS}

An initial assessment of LVEF and LVEF changes are important for deciding treatment and predicting prognosis in HFpEF patients. In addition, several confounding factors are associated with LVEF changes in worsened HFpEF patients.

Acknowledgements The authors acknowledge the efforts of Kumiko Watanabe and Hitomi Kobayashi for their outstanding technical assistance.
Contributors AY and YT: making article, drafting the article and conception of this study; YS and YK: performing statistical analysis; MT, TY, SA, TM, TS, MO, AK, TY and HK: obtaining general data; MO, AK and YT revising the article critically for important intellectual content.

Funding This study was supported in part by a grant-in-aid for Scientific Research (No. 16K09447) from the Japan Society for the Promotion of Science.

Competing interests None declared.

Patient consent for publication Obtained.

Ethics approval The study protocol was approved by the ethical committee of Fukushima Medical University, the investigation conformed to the principles outlined in the Declaration of Helsinki, and reporting of the study conforms to Strengthening the Reporting of Observational Studies in Epidemiology (STROBE) along with references to STROBE and the broader EQUATOR guidelines.

Provenance and peer review Not commissioned; externally peer reviewed. Data availability statement Data are available on reasonable request.

Open access This is an open access article distributed in accordance with the Creative Commons Attribution 4.0 Unported (CC BY 4.0) license, which permits others to copy, redistribute, remix, transform and build upon this work for any purpose, provided the original work is properly cited, a link to the licence is given, and indication of whether changes were made. See: https://creativecommons.org/ licenses/by/4.0/.

ORCID iD

Akiomi Yoshihisa http://orcid.org/0000-0001-5574-6617

\section{REFERENCES}

1 Lund LH, Vedin O, Savarese G. Is ejection fraction in heart failure a limitation or an opportunity? Eur J Heart Fail 2018;20:431-2.

2 Katsi V, Georgiopoulos G, Laina A, et al. Left ventricular ejection fraction as therapeutic target: is it the ideal marker? Heart Fail Rev 2017;22:641-55.

3 Ponikowski P, Voors AA, Anker SD, et al. 2016 ESC guidelines for the diagnosis and treatment of acute and chronic heart failure: the task force for the diagnosis and treatment of acute and chronic heart failure of the European Society of cardiology (ESC) developed with the special contribution of the heart failure association (HFA) of the ESC. Eur Heart J 2016;37:2129-200.

4 Yancy CW, Jessup M, Bozkurt B, et al. 2017 ACC/AHA/HFSA focused update of the 2013 ACCF/AHA guideline for the management of heart failure: a report of the American College of Cardiology/American heart association Task force on clinical practice guidelines and the heart failure Society of America. Circulation 2017;136:e137-61

5 Yancy CW, Jessup M, Bozkurt B, et al. 2013 ACCF/AHA guideline for the management of heart failure: a report of the American College of cardiology Foundation/American heart association Task force on practice guidelines. J Am Coll Cardiol 2013;62:e147-239.

6 Redfield MM. Heart failure with preserved ejection fraction. $N$ Engl J Med 2016;375:1868-77.

7 Sharma K, Kass DA. Heart failure with preserved ejection fraction: mechanisms, clinical features, and therapies. Circ Res 2014;115:79-96.

8 Lupón J, Gavidia-Bovadilla G, Ferrer E, et al. Dynamic trajectories of left ventricular ejection fraction in heart failure. J Am Coll Cardiol 2018;72:591-601.

9 Jørgensen ME, Andersson C, Vasan RS, et al. Characteristics and prognosis of heart failure with improved compared with persistently reduced ejection fraction: a systematic review and meta-analyses. Eur J Prev Cardiol 2018;25:366-76.

10 Lupón J, Díez-López C, de Antonio M, et al. Recovered heart failure with reduced ejection fraction and outcomes: a prospective study. Eur J Heart Fail 2017;19:1615-23.

11 Kalogeropoulos AP, Fonarow GC, Georgiopoulou V, et al. Characteristics and outcomes of adult outpatients with heart failure and improved or recovered ejection fraction. JAMA Cardiol 2016;1:510-8.

12 Florea VG, Rector TS, Anand IS, et al. Heart failure with improved ejection fraction: clinical characteristics, correlates of recovery, and survival: results from the valsartan heart failure trial. Circ Heart Fail 2016;9:e003123.

13 Breathett K, Allen LA, Udelson J, et al. Changes in left ventricular ejection fraction predict survival and hospitalization in heart failure with reduced ejection fraction. Circ Heart Fail 2016;9:e002962. 
14 Basuray A, French B, Ky B, et al. Heart failure with recovered ejection fraction: clinical description, biomarkers, and outcomes. Circulation 2014;129:2380-7.

15 von Elm E, Altman DG, Egger M, et al. Strengthening the reporting of observational studies in epidemiology (STROBE) statement: guidelines for reporting observational studies. BMJ 2007;335:806-8.

16 Sato Y, Yoshihisa A, Kimishima Y, et al. Subclinical hypothyroidism is associated with adverse prognosis in heart failure patients. Can $J$ Cardiol 2018;34:80-7.

17 Levey AS, Coresh J, Greene T, et al. Using standardized serum creatinine values in the modification of diet in renal disease study equation for estimating glomerular filtration rate. Ann Intern Med 2006;145:247-54.

18 Miura S, Yoshihisa A, Takiguchi M, et al. Association of hypocalcemia with mortality in hospitalized patients with heart failure and chronic kidney disease. J Card Fail 2015;21:621-7.

19 Shimizu T, Yoshihisa A, Kanno Y, et al. Relationship of hyperuricemia with mortality in heart failure patients with preserved ejection fraction. Am J Physiol Heart Circ Physiol 2015;309:H1123-9.

20 Yoshihisa A, Takeishi Y. Sleep disordered breathing and cardiovascular diseases. J Atheroscler Thromb 2019;26:315-27.

21 Yoshihisa A, Suzuki S, Yamaki T, et al. Impact of adaptive servoventilation on cardiovascular function and prognosis in heart failure patients with preserved left ventricular ejection fraction and sleepdisordered breathing. Eur J Heart Fail 2013;15:543-50.

22 Iwaya S, Yoshihisa A, Nodera M, et al. Suppressive effects of adaptive servo-ventilation on ventricular premature complexes with attenuation of sympathetic nervous activity in heart failure patients with sleep-disordered breathing. Heart Vessels 2014;29:470-7.

23 Yoshihisa A, Takiguchi M, Shimizu T, et al. Cardiovascular function and prognosis of patients with heart failure coexistent with chronic obstructive pulmonary disease. J Cardiol 2014;64:256-64.

24 Nakamura Y, Kunii H, Yoshihisa A, et al. Impact of peripheral artery disease on prognosis in hospitalized heart failure patients. Circ $J$ 2015;79:785-93.

25 Tendera M, Aboyans V, Bartelink M-L, et al. Esc guidelines on the diagnosis and treatment of peripheral artery diseases: document covering atherosclerotic disease of extracranial carotid and vertebral, mesenteric, renal, upper and lower extremity arteries: the task force on the diagnosis and treatment of peripheral artery diseases of the European Society of cardiology (ESC). Eur Heart J 2011;32:2851-906.

26 Rudski LG, Lai WW, Afilalo J, et al. Guidelines for the echocardiographic assessment of the right heart in adults: a report from the American Society of echocardiography endorsed by the European association of echocardiography, a registered branch of the European Society of cardiology, and the Canadian Society of echocardiography. J Am Soc Echocardiogr 2010;23:685-713. quiz 786-8.

27 van Veldhuisen DJ, Linssen GCM, Jaarsma T, et al. B-Type natriuretic peptide and prognosis in heart failure patients with preserved and reduced ejection fraction. J Am Coll Cardiol 2013;61:1498-506.

28 Edelmann F, Stahrenberg R, Gelbrich G, et al. Contribution of comorbidities to functional impairment is higher in heart failure with preserved than with reduced ejection fraction. Clin Res Cardiol 2011;100:755-64.

29 Ho JE, Enserro D, Brouwers FP, et al. Predicting heart failure with preserved and reduced ejection fraction: the International collaboration on heart failure subtypes. Circ Heart Fail 2016;9:e003116.

30 Ho JE, Lyass A, Lee DS, et al. Predictors of new-onset heart failure: differences in preserved versus reduced ejection fraction. Circ Heart Fail 2013;6:279-86.

31 Tsuji K, Sakata Y, Nochioka K, et al. Characterization of heart failure patients with mid-range left ventricular ejection fraction-a report from the CHART-2 study. Eur J Heart Fail 2017;19:1258-69.

32 Dunlay SM, Roger VL, Weston SA, et al. Longitudinal changes in ejection fraction in heart failure patients with preserved and reduced ejection fraction. Circ Heart Fail 2012;5:720-6.

33 Senni M, Paulus WJ, Gavazzi A, et al. New strategies for heart failure with preserved ejection fraction: the importance of targeted therapies for heart failure phenotypes. Eur Heart J 2014;35:2797-815.

34 Kotecha D, Lam CSP, Van Veldhuisen DJ, et al. Heart Failure With Preserved Ejection Fraction and Atrial Fibrillation: Vicious Twins. J Am Coll Cardiol 2016;68:2217-28.

35 Samson R, Jaiswal A, Ennezat PV, et al. Clinical phenotypes in heart failure with preserved ejection fraction. J Am Heart Assoc 2016;5. doi:10.1161/JAHA.115.002477

36 Miyata M, Yoshihisa A, Yamauchi H, et al. Impact of sleep-disordered breathing on myocardial damage and metabolism in patients with chronic heart failure. Heart Vessels 2015;30:318-24.

37 Yoshihisa A, Suzuki S, Yamauchi H, et al. Beneficial effects of positive airway pressure therapy for sleep-disordered breathing in heart failure patients with preserved left ventricular ejection fraction. Clin Cardiol 2015;38:413-21.

38 Mentz RJ, Kelly JP, von Lueder TG, et al. Noncardiac comorbidities in heart failure with reduced versus preserved ejection fraction. $J$ Am Coll Cardiol 2014;64:2281-93. 